\title{
Predicting thermal instability in a closed loop pulsating heat pipe system
}

\author{
Ping-Hei Chen ${ }^{\mathrm{c}, *}$, Ya-Wei Lee ${ }^{\mathrm{a}}$, Tien-Li Chang ${ }^{\mathrm{b}}$ \\ a Ordnance Readiness and Development Center, Nantou 552, Taiwan \\ ${ }^{\mathrm{b}}$ Mechanical and Systems Research Laboratories, Industrial Technology Research Institute, Hsinchu 310, Taiwan

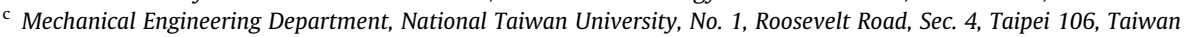

\section{A R T I C L E I N F O}

\section{Article history:}

Received 13 September 2007

Accepted 11 July 2008

Available online $\mathrm{xxxx}$

\section{Keywords:}

Pulsating heat pipe

Two-phase flow

MISO

NARMAX

\begin{abstract}
A B S T R A C T
Mathematical models for a closed loop pulsating heat pipe (CLPHP) with multiple liquid slugs and vapor plugs are presented in this study. The model considers the effect of thermal instability in different sections of a CLPHP at different operational conditions. Based on a neural network, an approach of nonlinear autoregressive moving average model with exogenous inputs (NARMAX) can be applied to the thermal instability of CLPHP. This study approximates the nonlinear behavior of CLPHP with a linear approximation method that can establish the relationship among the response temperature differences between evaporator, adiabatic, and condenser sections. A multi-input single-output (MISO) strategy is adopted in this study to approximate nonlinear behavior of CLPHP. The predicted results show that the effect of the three sections to vapor condensation could be precisely distinguished; meanwhile, thermal performance of CLPHP would be predicted. The development of nonlinear identification technique will be helpful to optimize and understand the heat transfer performance of thermal instability in the different designs of CLPHP.
\end{abstract}

(c) 2008 Elsevier Ltd. All rights reserved.

\section{Introduction}

Pulsating heat pipes (PHPs) are passive two-phase thermal control devices that were first introduced by Akachi [1]. A PHP is a special type of heat pipe whose driving force is the slug/plug motion of the working fluid in the tube, generated by evaporation. Several applications exist for PHPs, from electronics and structural thermal control to microgravity thermal control. The simple construction, lightweight, and low cost of PHPs also make them useful in space radiators, producing more isothermal characteristics.

Researchers have conducted many experiments to visualize flow patterns in an attempt to demonstrate the operational mechanism of PHPs. Results have shown that slug flow is the dominant flow pattern in a PHP [2]. Lee et al. reported that bubble oscillation is caused by nucleate boiling and vapor oscillation, and departures of small bubbles are considered to be the representative flow pattern at the evaporator and the adiabatic section [3]. Yang et al. found that the working fluid in a PHP pulsates intermittently at low power levels and flows in a single direction at high heat loads [4]. The results also indicate the relation between heat transfer rate and charging ratio, cooling method, and relative position of heating and cooling, etc. In another study, Khandekar et al. found that PHPs are essentially nonequilibrium heat transfer devices [5]. Liquid and vapor slug transport is caused by a fully thermally driven system. Because of the construction inherent in the device, no external

\footnotetext{
* Corresponding author. Tel.: +886 2 33662689; fax: +886 23670781/31755.

E-mail address: phchen@ntu.edu.tw (P.-H. Chen).
}

mechanical power source is required for fluid transport as in the case of DREAM pipes [6].

Nonlinear system analysis and prediction have been important topics in prior research. Related theories have also been developed and applied to a large class of nonlinear systems based on the functional series representations of Volterra and Wiener [7]. Volterra was the first to formulate mathematical expressions for an integral series by means of higher degree kernels, and Wiener showed how a related series could be orthogonalized. Billings and Voon [8] thoroughly reviewed early works on the Volterra and Wiener kernels nonlinear system identification approach.

Following the work of Wiener, a great deal of research has been conducted on efficiently computing the kernels of physical systems $[9,10]$. Results on kernel based identification techniques have been published recently [11-13] which model single-input singleoutput (SISO) systems. However, these models received little attention in contrast with the of multi-input multi-output (MIMO) Volterra model. Marmarelis [14] briefly introduced early works on multi-input Volterra modeling and Westwick and Kearney recently modeled a multi-input system using the Wiener theory [15].

The problem of two-phase flow oscillation in a closed loop pulsating heat pipe (CLPHP) is very complicated because it incorporates many unstable variables. Nowadays, no sufficient theory or semi-empirical model can precisely describe such dynamic behavior. The present study introduces the nonlinear autoregressive moving average model with exogenous inputs (NARMAX) modeling technique [16-18] in an attempt to estimate appropriate models for describing and predicting such unstable systems. New 


$\begin{array}{ll}\text { Nomenclature } \\ \text { Nomenclature } \\ c & \text { NARMAX model coefficients } \\ d & \text { system time delay } \\ E & \text { cost function } \\ F & \text { nonlinear function } \\ f & \text { frequency } \\ g & \text { auxiliary model coefficients } \\ k & \text { time } \\ m & \text { number of input } \\ r & \text { number of output } \\ n & \text { time lag } \\ s & \text { auxiliary model term } \\ w & \text { weighting function } \\ u & \text { system input } \\ y & \text { system output } \\ & \\ \text { Greek } & \text { symbols } \\ \varepsilon & \text { residue } \\ \alpha & \text { constant vector term }\end{array}$

$\begin{array}{ll}\delta & \text { Kronecker delta } \\ \theta & \text { white noise parameter vector } \\ \tau & \text { time lag }\end{array}$

Superscript

$i \quad$ ith row of Eq. (1)

$\ell \quad$ degree of nonlinearity

$\begin{array}{ll}\text { Subscripts } \\ \text { MPO } & \text { model predicted output } \\ m & \text { number of input } \\ n & \text { order } \\ p & \text { order of input } \\ q & \text { order of output } \\ r & \text { number of output } \\ u & \text { system input } \\ y & \text { system output } \\ \varepsilon & \text { residue }\end{array}$

insights into CLPHP dynamics can be obtained by analyzing the model structures.

\section{System description}

Fig. 1 illustrates the CLPHP used in the present study, with an overall width of $17 \mathrm{~cm}$ and height of $30 \mathrm{~cm}$. Herein the CLPHP is fabricated from a long copper capillary tube with an outside diameter of $4 \mathrm{~mm}$ and an inside diameter of $2 \mathrm{~mm}$. The bending radius of the " $U$ " bend in the evaporator section and the inverted " $U$ " bend in the condenser section are both $10 \mathrm{~mm}$, forming 10 snake-shaped CLPHP structures. The CLPHP includes evaporator, adiabatic and condenser sections. In the evaporator section, the thermal load is applied by $\mathrm{Ni}-\mathrm{Cr}$ alloy thermic wire with a width of $1.6 \mathrm{~mm}$. This wire is wrapped around the outer wall surface of the evaporator section at $2 \mathrm{~mm}$ intervals. Two axial fans set at a constant speed of $4500 \pm 100 \mathrm{rpm}$ are used as heat sinks.

The CLPHP operates at a fixed position in vertical bottom heating mode. Below the heat sink, the capillary tube is well heat-insulated by a thick heat insulation material that forms the adiabatic and evaporator sections. The heat received in the evaporator section is almost completely dissipated to the condenser section. A steady oscillation state can thus be sustained. In all, four fine T-type thermocouples with a diameter of $0.1 \mathrm{~mm}$ are attached to the outer wall surface of the capillary tube with conductive aluminum tape. These thermocouples cover exactly half of the CLPHP. This is necessary to provide information about the entire CLPHP due to the configuration symmetry at thermocouple T1. Thermocouples are numbered from T1 to T4; T1 and T2 are located in the condenser section while T3 and T4 are located in the evaporator section. Spacing between the neighboring thermocouples is $5 \mathrm{~cm}$ in the evaporator section and $10 \mathrm{~cm}$ in the condenser section. The temperature difference of condenser section is defined as $\Delta T_{h}\left(\Delta T_{h}=\mathrm{T} 2-\mathrm{T} 1\right)$, the temperature difference of adiabatic section is defined as $\Delta T_{\mathrm{a}}\left(\Delta T_{\mathrm{a}}=\mathrm{T} 3-\mathrm{T} 2\right)$, and the temperature difference of evaporator section is defined as $\Delta T_{\mathrm{c}}\left(\Delta T_{\mathrm{C}}=\mathrm{T} 4-\mathrm{T} 3\right)$.

This study uses a high-speed data acquisition system (MX100, Yokogawa) with four channels and a power supply unit. The data acquisition system is used to measure the thermal oscillation at the capillary tube wall surface using a sampling rate of $4 \mathrm{~Hz}$. The capillary tube, with a total length of $350 \mathrm{~cm}$, is bent to form five parallel channels. The thermocouples are carefully calibrated using a constant-temperature bath with an accuracy of $\pm 0.1^{\circ} \mathrm{C}$. The power supply has an error rate of less than $\pm 1 \%$.

In this study, the test heating powers are $20 \mathrm{~W}, 60 \mathrm{~W}$, and $100 \mathrm{~W}$. De-ionized (DI) water is the working fluid and the charge ratio is $60 \%$. Note that DI water is water containing no other ions except $\mathrm{H}_{3} \mathrm{O}^{+}$and $\mathrm{OH}^{-}$after a purification process and the charge ratio is defined as the ratio between the charged volume of working fluid and the total volume inside the CLPHP. According to tested results on the CLPHP over a wide range of charge ratio from $10 \%$ to $90 \%$ [19], the case with a charge ratio around $60 \%$ has the best thermal performance.

The CLPHP is under vacuum before being charged with working fluid, and is able to hold a vacuum level of $10^{-3}$ Torr. The maximum evaporation section temperature is limited to $150{ }^{\circ} \mathrm{C}$, which results in tests with heat loads up to $100 \mathrm{~W}$. All the experimental tests are performed on a heat load profile basis, with the intention to evaluate CLPHP behavior related to the applied heating power. All data points are chosen from a specific 500 -s period under normal CLPHP operating conditions, i.e. the CLPHP is close to a quasisteady state.

To better understand the cause for dynamic temperature responses at the condenser section of a heat pipe, this study used a glass closed-loop pulsating heat pipe (CLPHP) in visualization experiments under copper CLPHP operating conditions. The dimensions of the glass and copper CLPHPs are the same except that the wall of the glass CLPHP is twice as thick as the copper CLPHP. Although copper has a significantly higher thermal conductivity than glass, similar flow patterns in both heat pipes can be expected when the fluid inside experiences the same heat flux on the inner wall of the heat pipe. This study employs a high speed camera (XS-4, X-stream ${ }^{\mathrm{TM}}$, IDT, USA) with a sampling rate of 300 frames per second to capture flow patterns in a glass CLPHP at different heating powers. As Fig. 1 shows, the flow pattern is observed at the second loop of the CLPHP.

\section{The NARMAX approach}

A wide class of discrete-time multi-variable nonlinear stochastic systems can be represented by the NARMAX model under appropriate assumptions. In this study, a multi-input multi-output (MIMO) NARMAX model is derived from the radial basis function (RBF) neural network, as shown in Fig. 2. The RBF neural network 


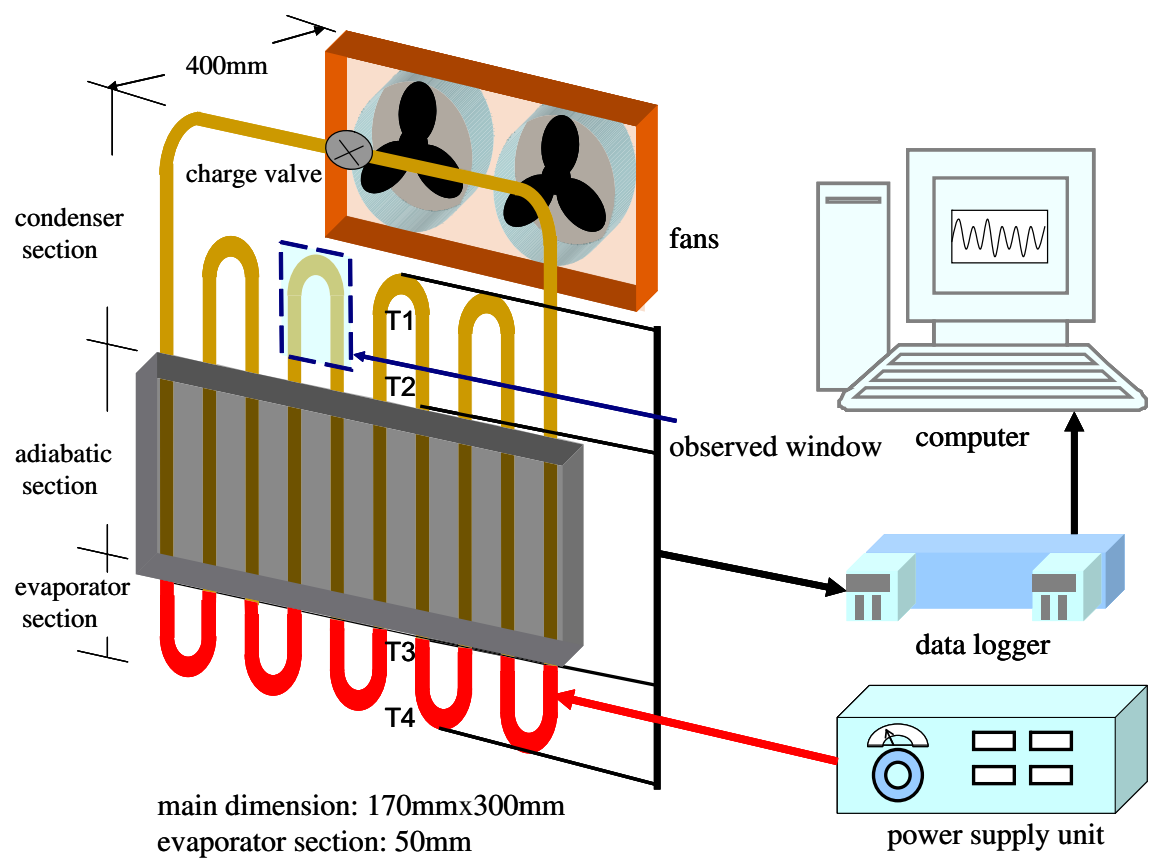
adiabatic section: $150 \mathrm{~mm}$ condenser section : $100 \mathrm{~mm}$

Fig. 1. Schematic view of experimental setup.

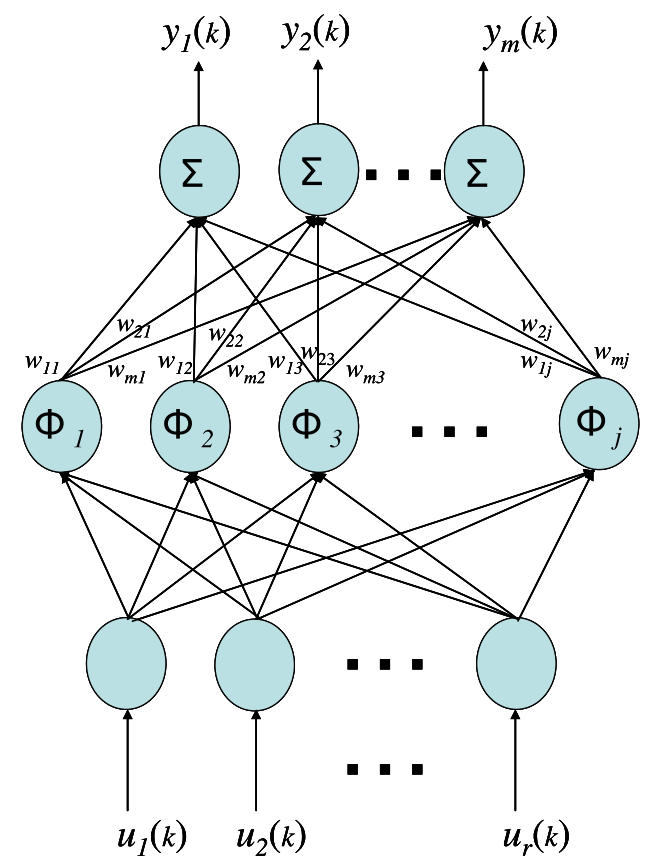

Hidden layer

Input layer

Fig. 2. Schematic view of a multi-input multi-output (MIMO) radial basis function neural network (RBFNN).

consists of an input layer with $r$ nodes, a hidden layer with $j$ neurons and an output layer with $m$ nodes. Input and output nodes can be presented in vector forms as $\vec{u}(k)=\left[u_{1}(k) \cdots u_{r}(k)\right]$ and $\vec{y}(k)=\left[y_{1}(k) \cdots y_{m}(k)\right]$, respectively. Each of the input nodes is connected to all the neurons in the hidden layer with the same unity weight. However, each of the hidden layer nodes is connected to the output node through different weights. For instance, the $m$ th output node is connected to all nodes in the hidden layer with different weighting, represented by $\vec{w}_{m}(k)=\left[w_{m 1}(k) \cdots w_{m j}(k)\right]$.
In short, an m-output $r$-input system can be described as follows:

$\underline{y}(k)=\underline{\alpha}+\underline{F^{\ell}}\left[\underline{y}(k-1), \ldots, \underline{y}\left(k-n_{y}\right), \underline{u}(k-d), \ldots\right.$,

$\left.\underline{u}\left(k-d-n_{u}\right), \underline{\varepsilon}(k-1), \ldots, \underline{\varepsilon}\left(k-n_{\varepsilon}\right)\right]+\underline{\varepsilon}(k)$

where

$\underline{y}(k)=\left[\begin{array}{c}y_{1}(k) \\ \vdots \\ y_{m}(k)\end{array}\right], \quad \underline{u}(k)=\left[\begin{array}{c}u_{1}(k) \\ \vdots \\ u_{r}(k)\end{array}\right], \quad \underline{\varepsilon}(k)=\left[\begin{array}{c}\varepsilon_{1}(k) \\ \vdots \\ \varepsilon_{m}(k)\end{array}\right]$

where $\underline{y}(k), \underline{u}(k)$ and $\underline{\varepsilon}(k)$ represent the system output, input and prediction errors, respectively, $d$ is the system time delay, $\ell$ is the degree of nonlinearity, $\underline{\alpha}$ is a constant vector term to accommodate mean levels, and $\underline{F}[\cdot]$ is a vector valued nonlinear function. Taking the $i^{\text {th }}$ row from Eq. (1) with different values of the maximum lag for each output, input, and noise gives

$$
\begin{aligned}
& y_{i}(k)=\alpha+F_{i}^{l}\left[y_{1}(k-1), \ldots, y_{1}\left(k-n_{y 1}^{i}\right), \ldots, y_{m}(k-1), \ldots, y_{m}\left(k-n_{y m}^{i}\right),\right. \\
& u_{1}(k-d), \ldots, u_{1}\left(k-d-n_{u 1}^{i}\right), \ldots, u_{r}(k-d), \ldots, u_{r}\left(k-d-n_{u r}^{i}\right), \\
& \varepsilon_{1}(k-1), \ldots, \varepsilon_{1}\left(k-n_{\varepsilon}\right), \ldots, \varepsilon_{1}\left(k-n_{\varepsilon}^{i}\right), \ldots, \varepsilon_{m}(k-1), \ldots, \\
& \left.\quad \varepsilon_{m}\left(k-n_{\varepsilon m}^{i}\right)\right]+\varepsilon_{i}(k), \quad k=1, \ldots, m
\end{aligned}
$$

This study employed a neural network approach to obtain the values of elements in both vectors $\underline{F}[\cdot]$ and $\underline{\alpha}$.

Tsang and Billings [20] used an orthogonal estimator to identify NARMAX models and assessed the statistical validity of the functional relationship between the different regions using structural equation modeling (SEM). An orthogonal estimator is a simple and efficient algorithm that allows each coefficient in the model to be estimated. At the same time, it indicates the contribution that each term makes to the system output, using the error reduction ratio (ERR). The ERR can be defined as

$\mathrm{ERR}_{i}=\frac{\sum_{k=1}^{N} g_{i}^{2} s_{i}^{2}(k)}{\sum_{k=1}^{N} y^{2}(k)} \times 100 \%$ 
where $g_{i}$ represents the coefficients and $s_{i}$ represents the terms of an auxiliary model constructed in such a way that the terms $s_{i}$ are orthogonal to the data records.

In this study, terms in the structural equation modeling approach represent combined and individual measured temperature differences at three sections of CLPHP at time steps earlier than the present time step $k$. A forward-regression algorithm is employed at each step to select the term with the highest ERR. In other words, the algorithm selects the term that contributes most to the reduction of the residual variance.

Together with the parameter estimation, the ERR value can be computed to indicate the significance of each term. A greater ERR value signifies a more important difference parameter. The terms can then be ranked according to their contribution to the overall mean squared prediction error.

Two strategies to evaluate model correctness and validity are indispensable once the significant terms have been identified and the associated parameter values have been estimated. If validation shows that the model is not good, some of the estimation design variables should be changed and the identification procedure should be iterated. Three different steps are iterated for model validation in this study and described as follows:

1. One-step prediction tests: the one-step prediction of the output is defined as

$$
\begin{aligned}
& \tilde{y}(k)=\widetilde{F}^{\ell}\left[y(k-1), \ldots, y\left(k-n_{y}\right), u(k-d), \ldots,\right. \\
& \left.u\left(k-d-n_{u}\right), \varepsilon(k-1, \tilde{\theta}), \ldots, \varepsilon\left(k-n_{\varepsilon}, \tilde{\theta}\right)\right]
\end{aligned}
$$

where $\widetilde{F}[\cdot]$ is the estimate of $F[\cdot], \tilde{\theta}$ is the parameter vector of the white noise, and $\varepsilon(k, \tilde{\theta})$ is the residue given by

$\varepsilon(k, \tilde{\theta})=y(k)-\tilde{y}(k)$

This is a by-product of the regression process. This prediction is obtained from the neural network through real historical measurements. The prediction is easily obtained from Eq. (5) by subtracting the prediction errors from the original data. One-step prediction provides short-term forecasts. The model is effectively reset at each step by inserting the appropriate values of the right hand side in Eq. (5). Any error is therefore reset at each step.

2. Correlation tests: The approach includes computing the autocorrelation function of the residues and the cross-correlation functions that can be existed between the residues and the inputs. For the sake of extending to nonlinear models, practical tests must be available to check the presence of nonlinearity in both raw time series and residues from the fitted models. Many tests have been proposed, but using higher-order correlation functions for validity of nonlinear systems has been successful.When the system is nonlinear, the residues should be unpredictable from all linear and nonlinear combinations of past inputs and outputs. This will be true if the following correlation tests are satisfied:

$\phi_{\varepsilon \varepsilon}(\tau)=\delta(\tau), \quad \phi_{u_{i} \varepsilon}(\tau)=0 \forall \tau$,

$\phi_{\left(u_{i} u_{j}\right)^{\prime} \varepsilon}(\tau)=0 \forall \tau, \quad \phi_{\left(u_{i} u_{j}\right)^{\prime} \varepsilon^{\prime}}(\tau)=0 \forall \tau$

$\phi_{\varepsilon^{2} u_{i}}(\tau)=0 \forall \tau \geqslant 0$

where $\phi_{a b}(\tau)=E[a(k-\tau) b(k)], \delta(\tau)$ is the Kronecker delta, $u(\tau)$ and $\varepsilon(\tau)$ are the input and the residues sequence, respectively, and the single quote indicates that the mean has been removed. The values of cross-correlated functions must lie between -0.05 and 0.05 .

3. Model predicted output tests: The model predicted output (MPO) providing long-term forecasts is a multi-step prediction, which is defined as

$$
\begin{aligned}
& \tilde{y}_{\mathrm{MPO}}(k)=\tilde{F}^{\ell}\left[\tilde{y}(k-1), \ldots, \tilde{y}\left(k-n_{y}\right), u(k-d), \ldots,\right. \\
& \left.u\left(k-d-n_{u}\right), 0, \ldots, 0\right]
\end{aligned}
$$

where the measured inputs are used to generate the model output. The zeros are present because the prediction errors will not be available when using the model to predict the output. An essential condition for accepting the model that the estimated model predicted outputs are in good agreement with the measured outputs. A comparison of measured and predicted results is provided, and the mean relative error (MRE) illustrates the accuracy of the tests.

This study uses multi-input single-output (MISO) black-box systems to estimate CLPHP nonlinearities. In this study, the inputs are the temperature differences at three sections during prior time steps, and the output is the temperature difference at the condenser section during the present time step. The nonlinearity of CLPHP is expressed by relating the predicted value of $\Delta T_{\mathrm{c}}(k)$ at the present time step with prior measured temperature differences at three separate sections of the CLPHP in Eq. (8). The structure equation modeling approach can identify which structural term has a greater contribution to $\Delta T_{\mathrm{c}}(k)$. Listing the order of importance on $\Delta T_{\mathrm{c}}(k)$ for these structural terms (the combinations of prior measured temperature differences or individual prior measured temperatures at three separate sections) helps to identify the thermal performances of the CLPHP tested in this study.
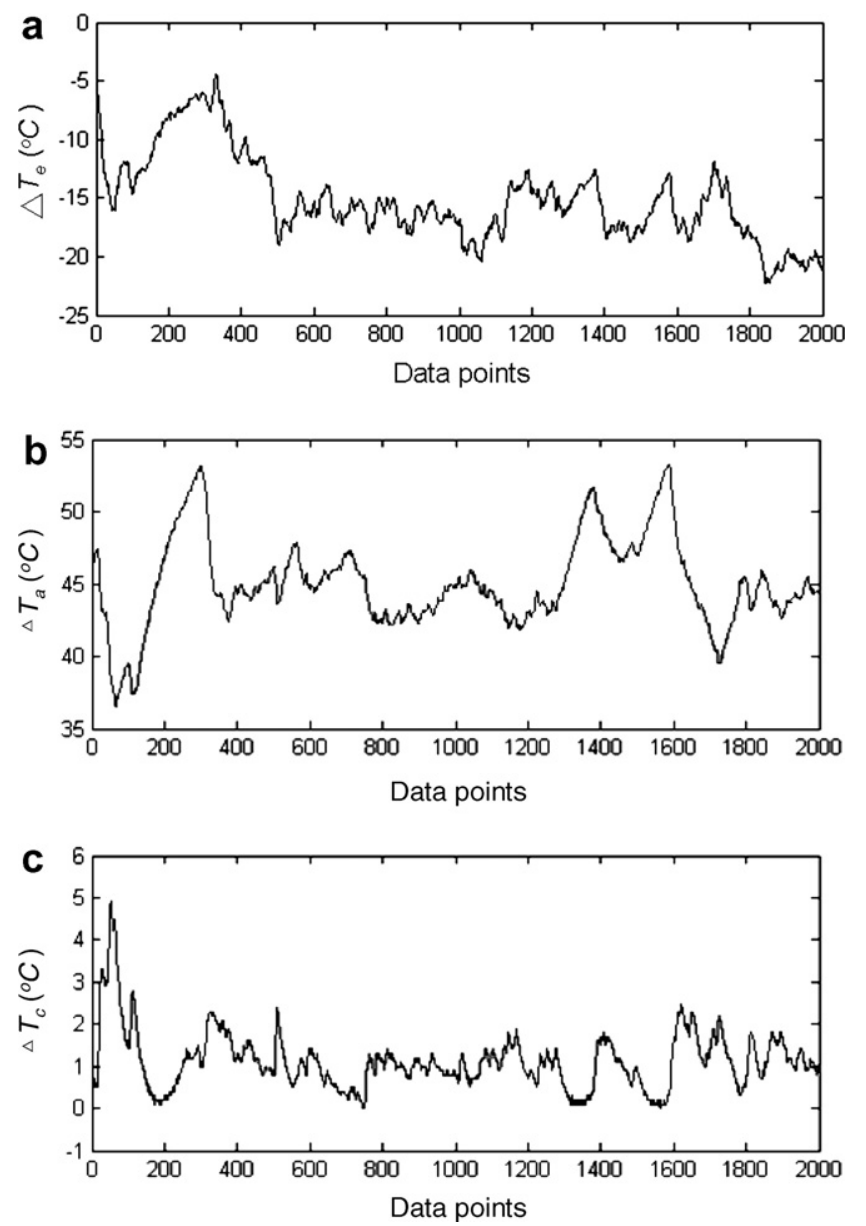

Fig. 3. I/O data points selected during a 500 s period at $20 \mathrm{~W}$ heating power: (a) the temperature difference at the evaporator section, $\Delta T_{\mathrm{e}}$ as input data 1 , (b) the temperature difference at the adiabatic section, $\Delta T_{\mathrm{a}}$ as input 2, and (c) the temperature difference at the condenser section $\Delta T_{\mathrm{c}}$ as output. 


\section{Results and discussions}

Figs. 3-5 show the measured data points for different operational conditions. These data points represent the wall temperature differences of the evaporator $\left(\Delta T_{\mathrm{e}}\right)$, adiabatic $\left(\Delta T_{\mathrm{a}}\right)$, and condenser sections $\left(\Delta T_{\mathrm{c}}\right)$ composing a single CLPHP tube. As the heating power increases, the three wall temperature differences behave correspondingly. That is, flow oscillations occur more likely at the three CLPHP sections at higher heating powers. Fig. 4 indicates that the measured results for $60 \%$ charge ratio and $100 \mathrm{~W}$ heating power show more severe variations than other operating conditions. Clearly, $\Delta T_{\mathrm{e}}$ and $\Delta T_{\mathrm{a}}$ show similar temperature responses, and $\Delta T_{\mathrm{c}}$ exhibits an exactly opposite response. This implies that CLPHP flow oscillations are resonant at all three sections under these operational conditions.

This study also considers quadratic terms in NARMAX models, and each model is designed for 12 terms. The appropriate maximum time lags are set as 10 for both inputs and output, and set as 0 in residue to avoid computation problems. Under this assumption, every predicted output is simulated by the same initial conditions. That is, the model structure candidates are combinations of 20 input signals and 10 output signals.

The estimated temperature difference at the condenser section, $\Delta T_{\mathrm{c}}(k)$, are obtained from measured input data using the model predicted output (MPO) approach mentioned in Section 3. Tables 1-3 list the coefficients and ERR values of structural terms at heating powers of $20 \mathrm{~W}, 60 \mathrm{~W}$, and $100 \mathrm{~W}$, respectively. These tables
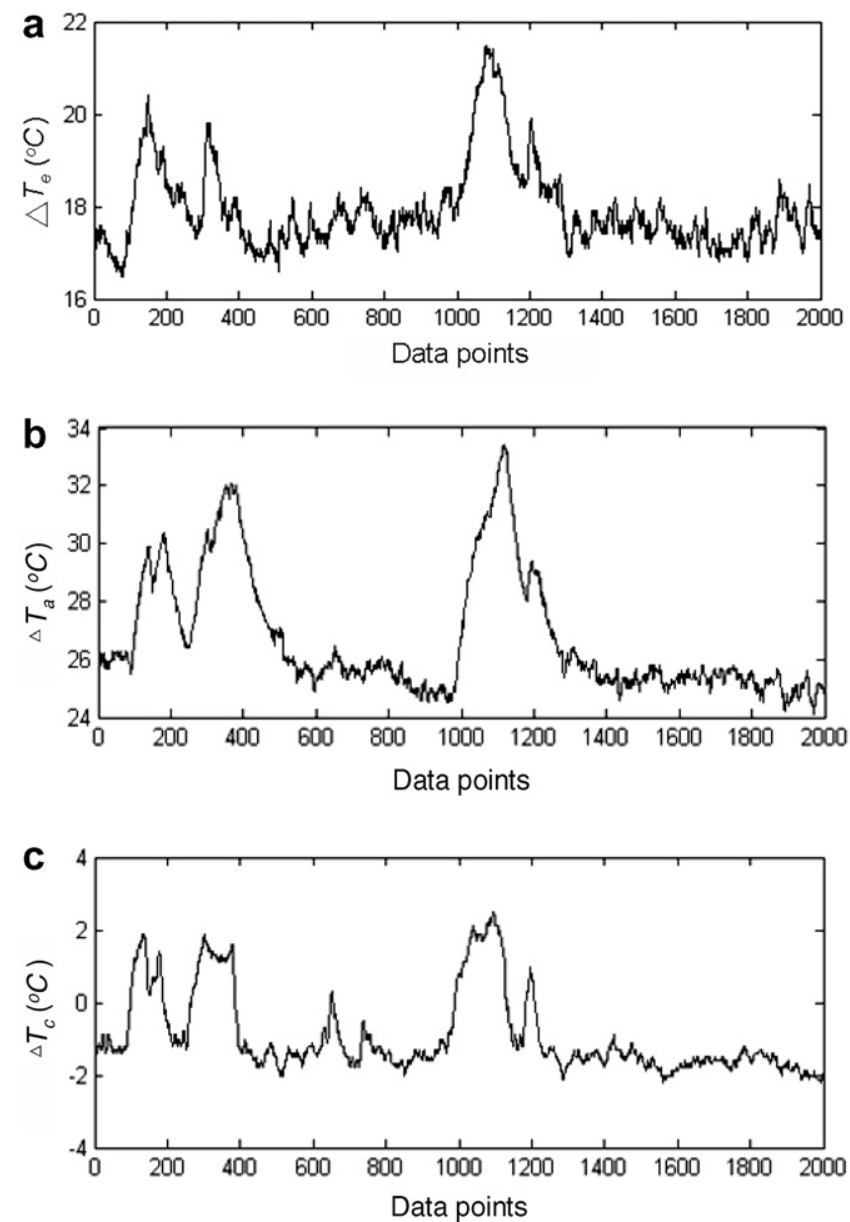

Fig. 4. I/O data points selected during a $500 \mathrm{~s}$ period at $60 \mathrm{~W}$ heating power: (a) the temperature difference at the evaporator section, $\Delta T_{\mathrm{e}}$ as input data 1 , (b) the temperature difference at the adiabatic section, $\Delta T_{\mathrm{a}}$ as input 2 , and (c) the temperature difference at the condenser section $\Delta T_{\mathrm{c}}$ as output.
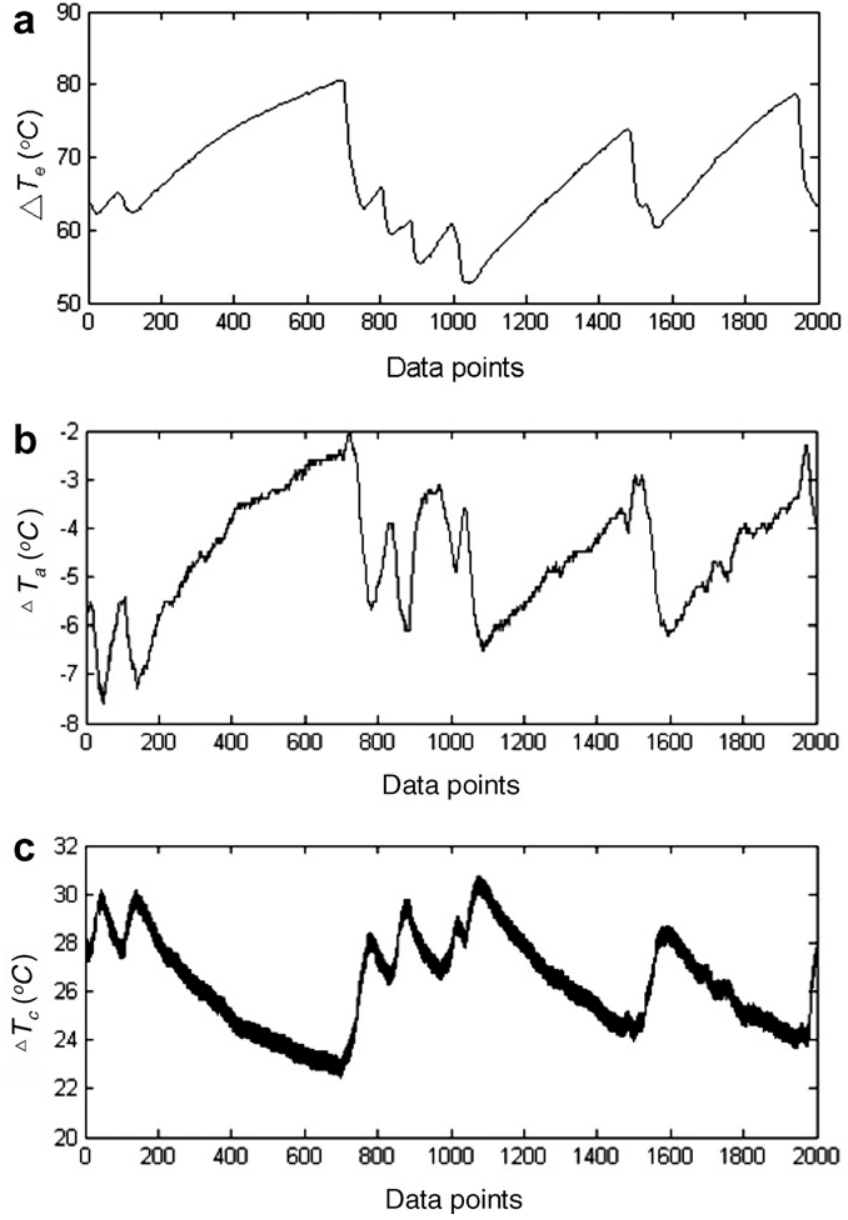

Fig. 5. $\mathrm{I} / \mathrm{O}$ data points selected during a $500 \mathrm{~s}$ period at $100 \mathrm{~W}$ heating power: (a) the temperature difference at the evaporator section, $\Delta T_{\mathrm{e}}$ as input data 1 , (b) the temperature difference at the adiabatic section, $\Delta T_{\mathrm{a}}$ as input 2, and (c) the temperature difference at the condenser section $\Delta T_{\mathrm{c}}$ as output.

Table 1

Quadratic NARMAX model of $\Delta T_{\mathrm{c}}(k)$ for $60 \%$ charge ratio and $20 \mathrm{~W}$ heating power

\begin{tabular}{lcl}
\hline Structural term & Coefficient & ERR \\
\hline$\Delta T_{\mathrm{c}}(k-1)$ & 1.4698 & 0.5967 \\
$\Delta T_{\mathrm{c}}(k-4)$ & -1.3278 & 0.2002 \\
$\Delta T_{\mathrm{c}}(k-2)$ & 0.0986 & $0.9970 \mathrm{E}-1$ \\
$\Delta T_{\mathrm{c}}(k-1) * \Delta T_{\mathrm{c}}(k-2)$ & 0.0006 & $0.8291 \mathrm{E}-1$ \\
$\Delta T_{\mathrm{c}}(k-2) * \Delta T_{\mathrm{c}}(k-2)$ & 0.0646 & $0.5852 \mathrm{E}-2$ \\
$\Delta T_{\mathrm{a}}(k-7) * \Delta T_{\mathrm{a}}(k-7)$ & -0.5756 & $0.4098 \mathrm{E}-2$ \\
$\Delta T_{\mathrm{a}}(k-3) * \Delta T_{\mathrm{a}}(k-9)$ & 0.0002 & $0.2471 \mathrm{E}-2$ \\
$\Delta T_{\mathrm{e}}(k-2) * \Delta T_{\mathrm{e}}(k-10)$ & -0.0283 & $0.2064 \mathrm{E}-2$ \\
$\Delta T_{\mathrm{a}}(k-1) * \Delta T_{\mathrm{a}}(k-9)$ & 0.0073 & $0.1889 \mathrm{E}-2$ \\
$\Delta T_{\mathrm{e}}(k-9) * \Delta T_{\mathrm{a}}(k-10)$ & 0.0287 & $0.1536 \mathrm{E}-2$ \\
$\Delta T_{\mathrm{c}}(k-1) * \Delta T_{\mathrm{c}}(k-6)$ & 1.5904 & $0.1472 \mathrm{E}-2$ \\
$\Delta T_{\mathrm{e}}(k-2) * \Delta T_{\mathrm{e}}(k-2)$ & 0.0050 & $0.1105 \mathrm{E}-2$ \\
\hline
\end{tabular}

list the structural terms according to their order of contribution to $\Delta T_{\mathrm{c}}$. Table 1 shows that the five most important terms are all combinations of measured values of $\Delta T_{\mathrm{c}}$ at prior time steps, which are $\Delta T_{\mathrm{c}}(k-1), \Delta T_{\mathrm{c}}(k-4), \Delta T_{\mathrm{c}}(k-2), \Delta T_{\mathrm{c}}(k-1) * \Delta T_{\mathrm{c}}(k-2)$, and $\Delta$ $T_{\mathrm{c}}(k-2) * \Delta T_{\mathrm{c}}(k-2)$. The total contribution of these five terms is 98.54\%. Model structure analysis shows that the condenser section has a great effect upon itself under $60 \%$ charge ratio and 20 W heating power operating conditions. The temperature difference at the adiabatic section is the next most important, while the evaporator section ranks is the least important. The model structure analysis results correspond to the flow dynamics of the tested CLPHP. A 
Table 2

Quadratic NARMAX model of $\Delta T_{\mathrm{c}}(k)$ at $60 \mathrm{~W}$ heating power

\begin{tabular}{lrl}
\hline Structural term & Coefficient & ERR \\
\hline$\Delta T_{\mathrm{c}}(k-1)$ & 1.0703 & 0.5873 \\
$\Delta T_{\mathrm{a}}(k-4)$ & -0.2161 & 0.1803 \\
$\Delta T_{\mathrm{a}}(k-4) * \Delta T_{\mathrm{c}}(k-9)$ & -0.3225 & 0.1101 \\
$\Delta T_{\mathrm{a}}(k-2)$ & 0.1842 & $0.3988 \mathrm{E}-1$ \\
$\Delta T_{\mathrm{a}}(k-3) * \Delta T_{\mathrm{a}}(k-10)$ & 0.0395 & $0.1898 \mathrm{E}-1$ \\
$\Delta T_{\mathrm{a}}(k-1) * \Delta T_{\mathrm{a}}(k-1)$ & -0.0087 & $0.1525 \mathrm{E}-1$ \\
$\Delta T_{\mathrm{c}}(k-1) * \Delta T_{\mathrm{c}}(k-1)$ & 0.0365 & $0.1156 \mathrm{E}-1$ \\
$\Delta T_{\mathrm{c}}(k-7) * \Delta T_{\mathrm{e}}(k-9)$ & 0.0152 & $0.9569 \mathrm{E}-2$ \\
$\Delta T_{\mathrm{c}}(k-3) * \Delta T_{\mathrm{a}}(k-7)$ & -0.0025 & $0.8761 \mathrm{E}-2$ \\
$\Delta T_{\mathrm{e}}(k-7) * \Delta T_{\mathrm{e}}(k-8)$ & -0.3264 & $0.7453 \mathrm{E}-2$ \\
$\Delta T_{\mathrm{c}}(k-7) * \Delta T_{\mathrm{e}}(k-1)$ & 0.1706 & $0.6345 \mathrm{E}-2$ \\
$\Delta T_{\mathrm{a}}(k-10) * \Delta T_{\mathrm{a}}(k-10)$ & -0.0153 & $0.4501 \mathrm{E}-2$ \\
\hline
\end{tabular}

Table 3

Quadratic NARMAX model of $\Delta T_{\mathrm{c}}(k)$ at $100 \mathrm{~W}$ heating power

\begin{tabular}{lcl}
\hline Structural term & Coefficient & ERR \\
\hline$\Delta T_{\mathrm{e}}(k-4)$ & 0.4234 & 0.5500 \\
$\Delta T_{\mathrm{e}}(k-8)$ & 0.5711 & 0.2003 \\
$\Delta T_{\mathrm{e}}(k-3) * \Delta T_{\mathrm{e}}(k-4)$ & 0.0025 & 0.1079 \\
$\Delta T_{\mathrm{e}}(k-10) * \Delta T_{\mathrm{e}}(k-1)$ & -0.1425 & $0.9472 \mathrm{E}-1$ \\
$\Delta T_{\mathrm{a}}(k-9)$ & -1.4257 & $0.2257 \mathrm{E}-1$ \\
$\Delta T_{\mathrm{e}}(k-9) * \Delta T_{\mathrm{a}}(k-10)$ & -0.1360 & $0.1150 \mathrm{E}-1$ \\
$\Delta T_{\mathrm{e}}(k-1) * \Delta T_{\mathrm{a}}(k-9)$ & -0.0042 & $0.5038 \mathrm{E}-2$ \\
$\Delta T_{\mathrm{e}}(k-1) * \Delta T_{\mathrm{a}}(k-2)$ & 0.0660 & $0.3262 \mathrm{E}-2$ \\
$\Delta T_{\mathrm{e}}(k-4) * \Delta T_{\mathrm{a}}(k-2)$ & 0.0603 & $0.1557 \mathrm{E}-2$ \\
$\Delta T_{\mathrm{e}}(k-4) * \Delta T_{\mathrm{a}}(k-8)$ & -0.0919 & $0.1172 \mathrm{E}-2$ \\
$\Delta T_{\mathrm{e}}(k-6) * \Delta T_{\mathrm{a}}(k-8)$ & 0.0978 & $0.1136 \mathrm{E}-2$ \\
$\Delta T_{\mathrm{c}}(k-1)$ & 2.3155 & $0.8162 \mathrm{E}-3$ \\
\hline
\end{tabular}

heating power of $20 \mathrm{~W}$ is too low to create a circulating two-phase flow in the CLPHP system, and merely cause the pool of working fluid to boil. A vapor bubble rises from the liquid column in the evaporator section and flows through the adiabatic section into the condenser section, as Fig. 6 shows. As the vapor bubble passes through the temperature measuring locations, it causes an oscillating temperature difference at the condenser section. However, vapor bubble generation is quite random. Therefore, the dynamic response of temperature difference in the condenser section is highly related to the size and flight time of the vapor bubble in the condenser section. Consequently, the temperature difference in the condenser section at any instant is closely correlated with prior measured temperature differences in the condenser section. Both evaporator and adiabatic sections have a much lesser effect on the predicted temperature difference in the condenser section than the condenser section.

For a scenario with $20 \mathrm{~W}$ heating power, the liquid phase of DI water occupies nearly all of the regions in both the evaporator and adiabatic sections. As the bubbles leave the pipe wall and rise in the liquid column in the evaporator section, the upper part of the evaporator section heats less water than the lower part. The measured temperature at the upper part of the evaporator section is greater than the lower part though the same amount of heat flux is applied to the water liquid at both locations. As a result, Fig. 3(a) shows negative measured values in $\Delta T_{\mathrm{e}}$. Fig. 3(b) shows that the temperature difference in the adiabatic section is large because one temperature probe is located in a region occupied mostly by water vapor and the other probe is located in a region occupied mostly by water liquid. Therefore, the temperature differences in both evaporator and adiabatic sections have far less effect on $\Delta T_{\mathrm{c}}(k)$ than that at the condenser section.

Table 2 lists the coefficient and the contribution of every structural term on $\Delta T_{\mathrm{c}}(k)$ for $60 \mathrm{~W}$ heating power. The term, $\Delta T_{\mathrm{c}}(k-1)$, is the most important term to $\Delta T_{\mathrm{c}}(k)$, and $\Delta T_{\mathrm{a}}(k-4)$, $\Delta T_{\mathrm{a}}(k-4) * \Delta T_{\mathrm{c}}(k-9), \Delta T_{\mathrm{a}}(k-2), \Delta T_{\mathrm{a}}(k-3) * \Delta T_{\mathrm{a}}(k-10)$ follow. The most significant term for $\Delta T_{\mathrm{c}}(k)$ is $\Delta T_{\mathrm{c}}(k-1)$, which is same for both $20 \mathrm{~W}$ and $60 \mathrm{~W}$ heat settings. The five most important terms comprise $93.66 \%$ of the total contributions to $\Delta T_{\mathrm{c}}(k)$. Because vapor bubbles are large enough to occupy the entire cross section of pipe when the heating power applied to the evaporator section increases from $20 \mathrm{~W}$ to $60 \mathrm{~W}$, they push the water column upwards. Segmented water slugs then travel in alternate directions at the adiabatic section, as Fig. 7 shows. The temperature difference in the condenser section is presumably affected by the size and speed of water slugs travelling through both adiabatic and condenser sections. In this case, the temperature difference in the adiabatic section has a greater effect on $\Delta T_{\mathrm{c}}(k)$ as the heating power increases. However, the evaporator section is still mostly occupied by liquid water. This can be confirmed by the small variation $\left(<5^{\circ} \mathrm{C}\right)$ in measured temperature difference at the evaporator section, as Fig. 4(a) indicates. Measured results in Fig. 4(a) and (b) indicate that the temperature difference varies more in the adiabatic section than the evaporator section.

Table 3 lists the coefficient and the contribution of every structural term on $\Delta T_{\mathrm{c}}(k)$ for $100 \mathrm{~W}$ heating power. Unlike the cases of low and medium heating powers, the four most important terms all consist of prior measured data of $\Delta T_{\mathrm{e}}$. In this heating condition, a

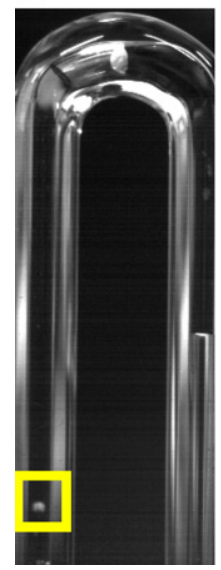

b

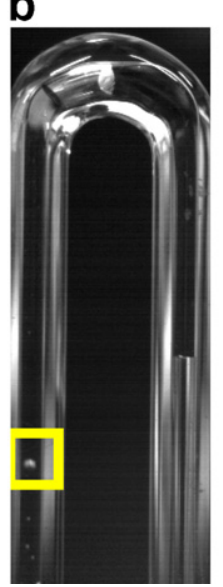

C

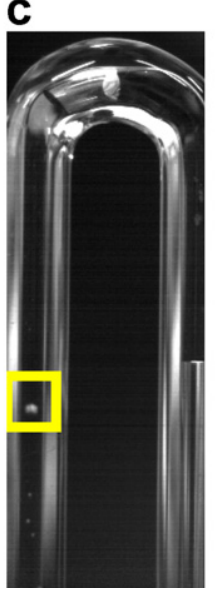

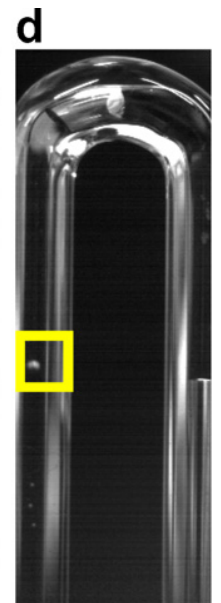

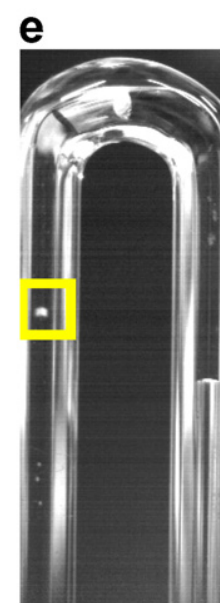

Fig. 6. Sequential photos showing CLPHP two-phase flow patterns in which a vapor bubble is tracked in the square box at $20 \mathrm{~W}$ heating power. 
a

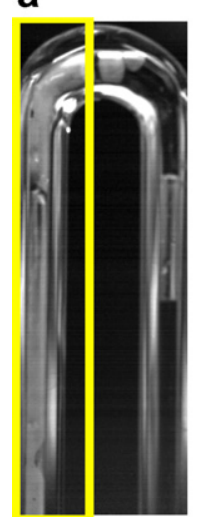

b

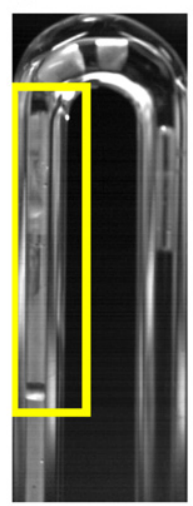

C

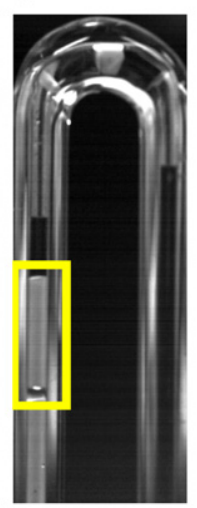

d

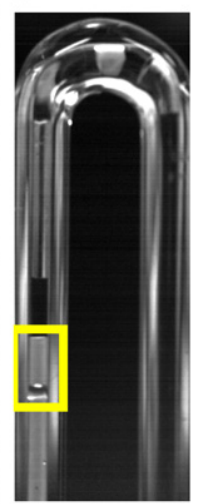

e

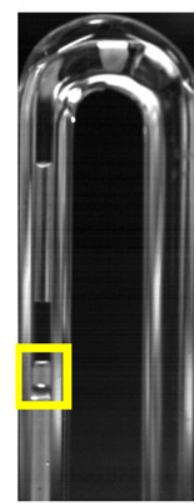

Fig. 7. Sequential photos showing CLPHP two-phase flow patterns in which a vapor-liquid transition phenomenon is tracked at $60 \mathrm{~W}$ heating power.

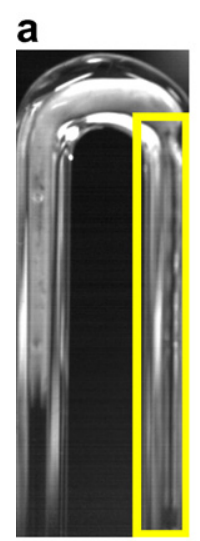

f

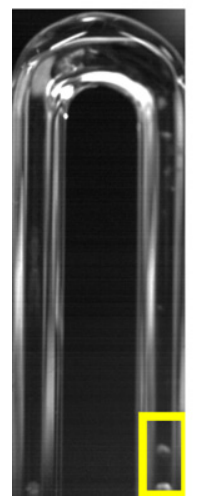

b

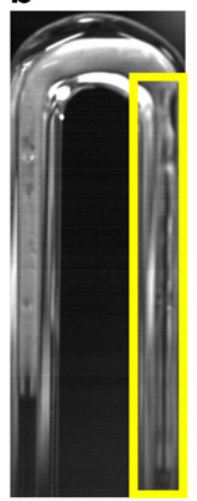

g

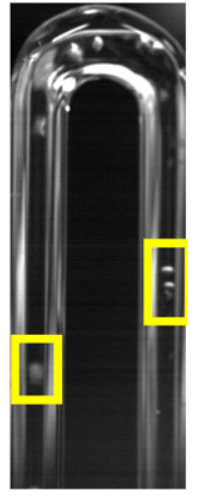

C

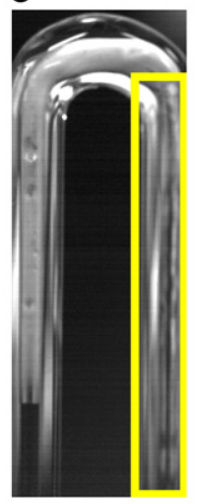

h

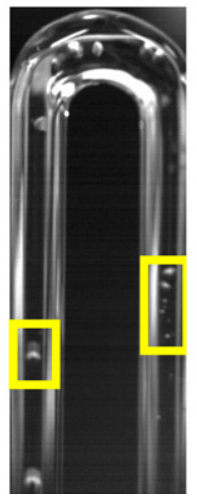

d

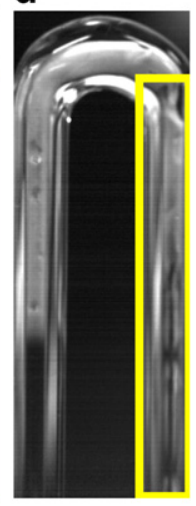

i

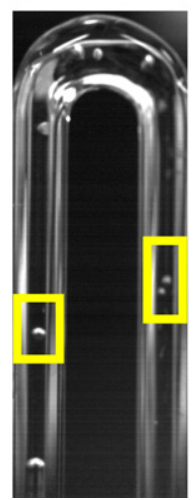

e

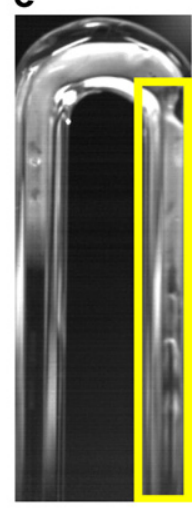

j

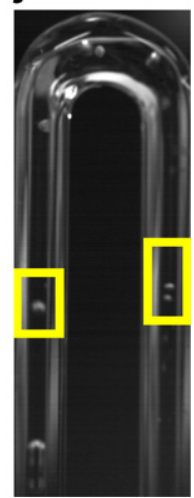

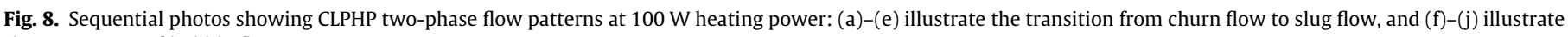
the occurrence of bubbly flow.

the heat flux is large enough to cause alternative flow patterns between the bubbly flow and slug/churn flow, as Fig. 8 shows. This high nonlinearity reveals that the effect of $\Delta T_{\mathrm{e}}$ on $\Delta T_{\mathrm{c}}$ is greatly enhanced as the heating power increases to $100 \mathrm{~W}$.

Fig. 9 compares the measured $\Delta T_{\mathrm{c}}(k)$ and model predicted $\Delta T_{c}(k)$ under $60 \%$ charge ratio and $20 \mathrm{~W}$ heating power conditions. This figure reveals that the model provides a range of relative error from -8.92 to +3.79 with $90 \%$ of the compared results. The mean relative error (MRE) for total compared results is 6.62\% (Fig. 10). Fig. 11 compares the measured $\Delta T_{\mathrm{c}}(k)$ and model predicted $\Delta T_{\mathrm{c}}(k)$ under $60 \%$ charge ratio and $60 \mathrm{~W}$ heating power conditions. Relative error under these conditions ranges from -32.50 to +9.64 with
$90 \%$ of the compared results. The mean relative error (MRE) is 22.14\% (Fig. 12) in all compared results.

The test results in Fig. 13 show that the model is reasonable, because the test results are nearly all inside the confidence bands (95\% confidence). Because only small deviations are present, this estimated model can be confirmed that it can precisely grasp the real dynamics of two-phase flow oscillation in a CLPHP.

Fig. 14 compares one-step prediction results with the measured results. The simulated time-series approximates the experimental data in a satisfactory way, and shows that flow oscillations occur. The MRE is $1.31 \%$ for the correlation between measured and prediction results of $\Delta T_{\mathrm{c}}$ one-time-step ahead 


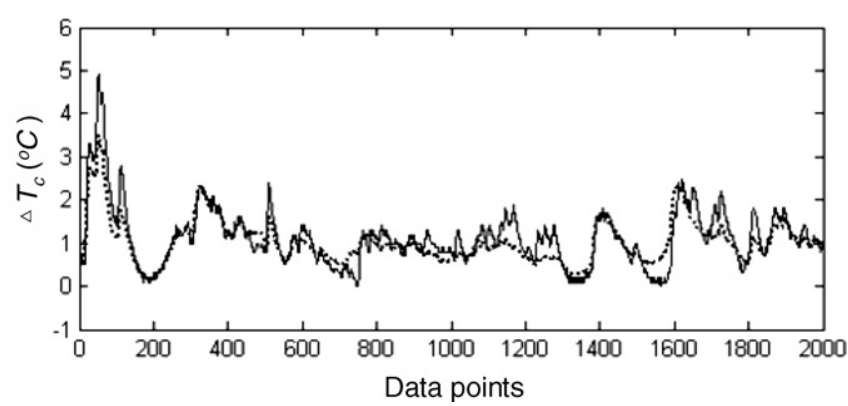

Fig. 9. Comparison between predicted results (dotted line) by the model predicted output (MPO) approach and measured results (solid line) at $20 \mathrm{~W}$ heating power.

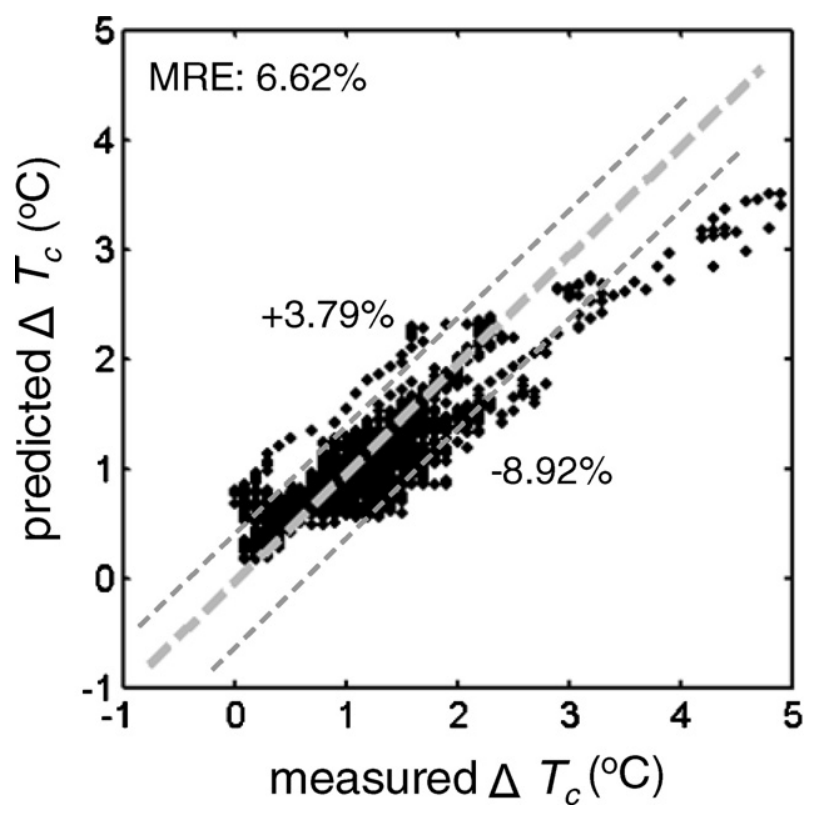

Fig. 10. Correlation between predicted results by the model predicted output (MPO) approach and measured results at $20 \mathrm{~W}$ heating power.

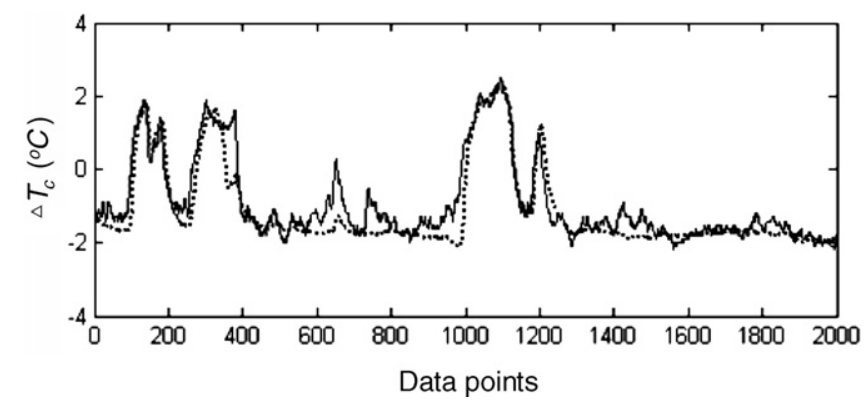

Fig. 11. Comparison between predicted results (dotted line) by the model predicted output (MPO) approach and measured results (solid line) at $60 \mathrm{~W}$ heating power.

(Fig. 15). Therefore, this result can be extended to the design of a real-time control system.

Fig. 16 shows the model predicted output by taking the model listed in Table 3 into the prediction process and comparing it with the same time series used for training. Some tiny deviations can be found in this prediction, but almost $90 \%$ of the compared results are within an acceptable error range of $\pm 3.42 \%$ (Fig. 17). The MRE

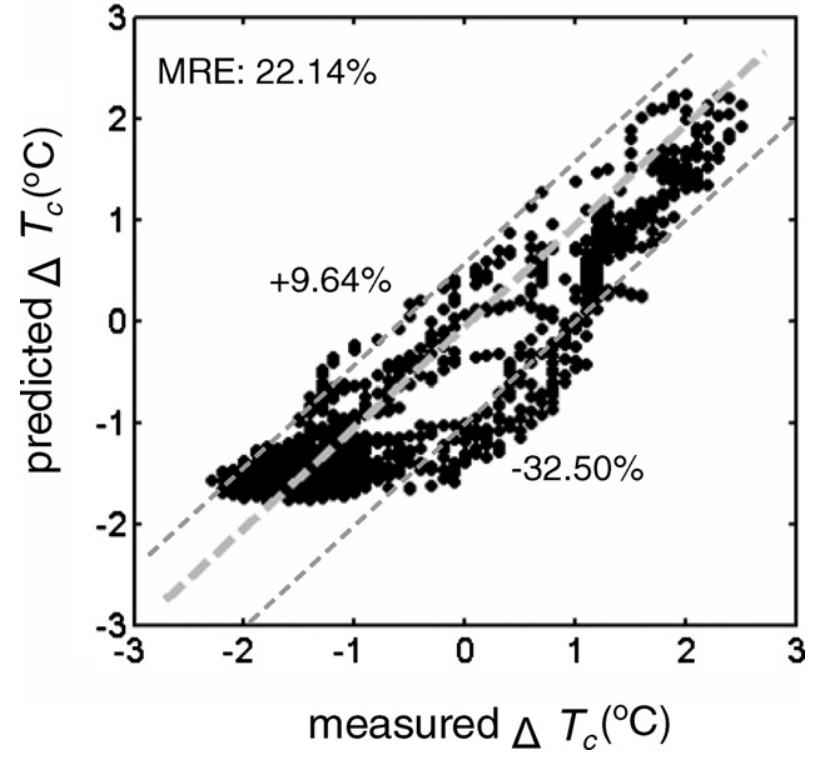

Fig. 12. Correlation between predicted results (dotted line) by the model predicted output (MPO) approach and measured results (solid line) at $60 \mathrm{~W}$ heating power.

is $3.54 \%$ for all compared results. Although this prediction contains a few deviations, this estimated model shows better simulated results than other methods, especially for such a long period prediction.

Note that a single model was successfully employed to predict the temperature difference at the condenser section under three separate heating conditions, low $(20 \mathrm{~W})$, medium $(60 \mathrm{~W})$, and high $(100 \mathrm{~W})$. However, the coefficients of structural terms in the model vary among various tested cases. The value of MRE at $60 \mathrm{~W}$ heating power is much greater than MRE values in both 20 and $100 \mathrm{~W}$ cases, as indicated by Figs. 10, 12, and 17. This low MRE value indicates that quasi-periodic temperature difference response, shown in Fig. 5(c) at $100 \mathrm{~W}$ heating power, can be better predicted by the proposed model than the appearance of random spikes in the temperature difference response, shown in Fig. 4 (c) at $60 \mathrm{~W}$. The same model was also successfully tested for scenarios with eight different heating powers between $20 \mathrm{~W}$ and $100 \mathrm{~W}$, but these results are not presented in the current manuscript. Furthermore, the thermal resistance of a CLPHP can be calculated by summing the measured temperature differences for all three sections.

\section{Conclusion}

Thermal instabilities generated by two-phase flow oscillations in the CLPHP are extremely unpredictable. In such nonlinear systems, physical properties interact in a complex way, and no rules can fully analyze their system dynamics. Unlike previous studies, this study successfully introduces the NARMAX approach to conduct a multi-variable investigation. This study also illustrates that there are reasonable agreements between predicted and measured temperature difference responses at the condenser section for heating power ranging from $20 \mathrm{~W}$ to $100 \mathrm{~W}$ at the evaporator section. The maximum value of mean relative error (MRE) between predicted and measured temperature difference responses at the condenser section is $22.14 \%$ and occurs at the test case with $60 \mathrm{~W}$.

Although the predicted results follow the measured results well, the physical interpretability of the model is lost. This is due 

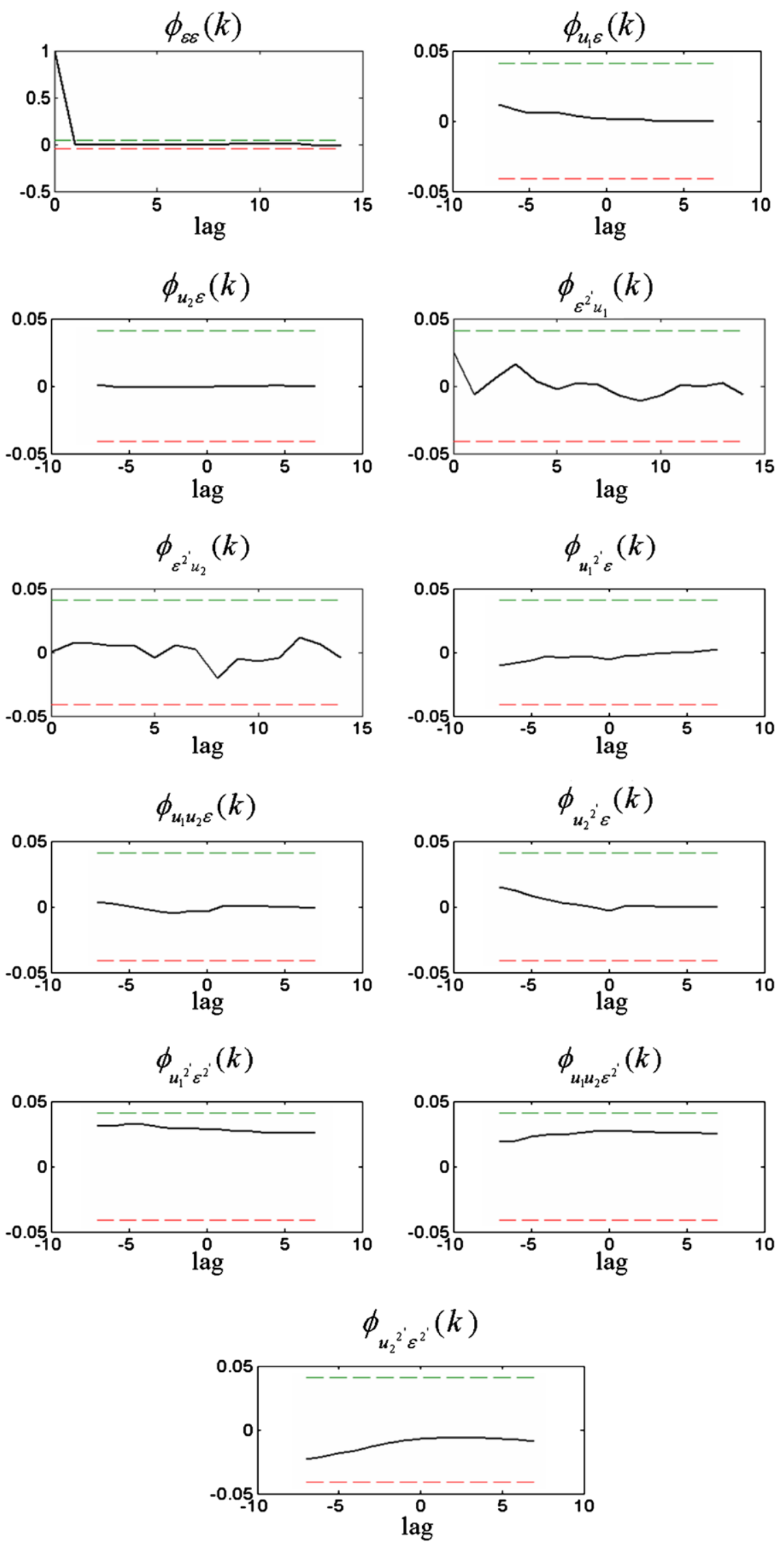

Fig. 13. Model correlation tests for the case at $100 \mathrm{~W}$ heating power. 


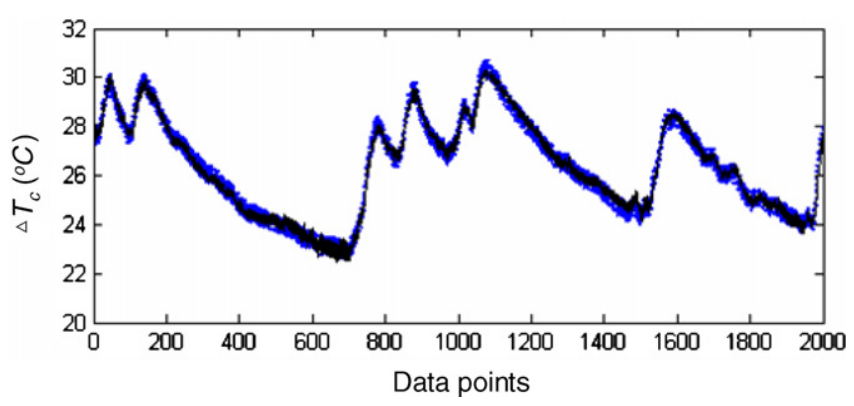

Fig. 14. Comparison between one-step predicted results (dotted line) and measured results (solid line) at $100 \mathrm{~W}$ heating power.

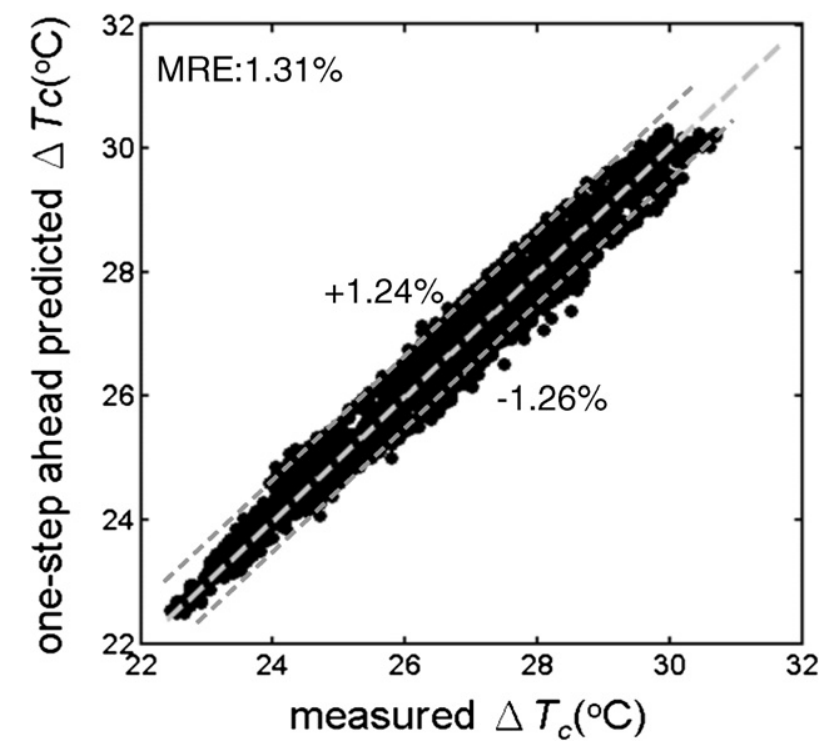

Fig. 15. Correlation between one-step predicted results and measured results at $100 \mathrm{~W}$ heating power.

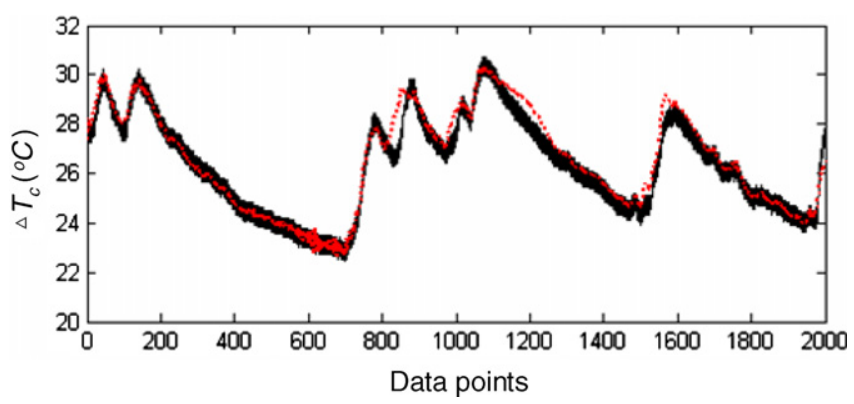

Fig. 16. Comparison between predicted results (dotted line) by the model predicted output (MPO) approach and measured results (solid line) at $100 \mathrm{~W}$ heating power.

to inherent problems with discrete-time estimation using bandlimited input signals and also to the great variability of model parameters when different nonlinear terms are included. Even though the temperature responses at different sections of the tested CLPHP are quite random, combining these identification techniques and time-domain NARMAX modeling can effectively capture two-phase flow oscillation in a CLPHP for the heating power from $20 \mathrm{~W}$ to $100 \mathrm{~W}$.

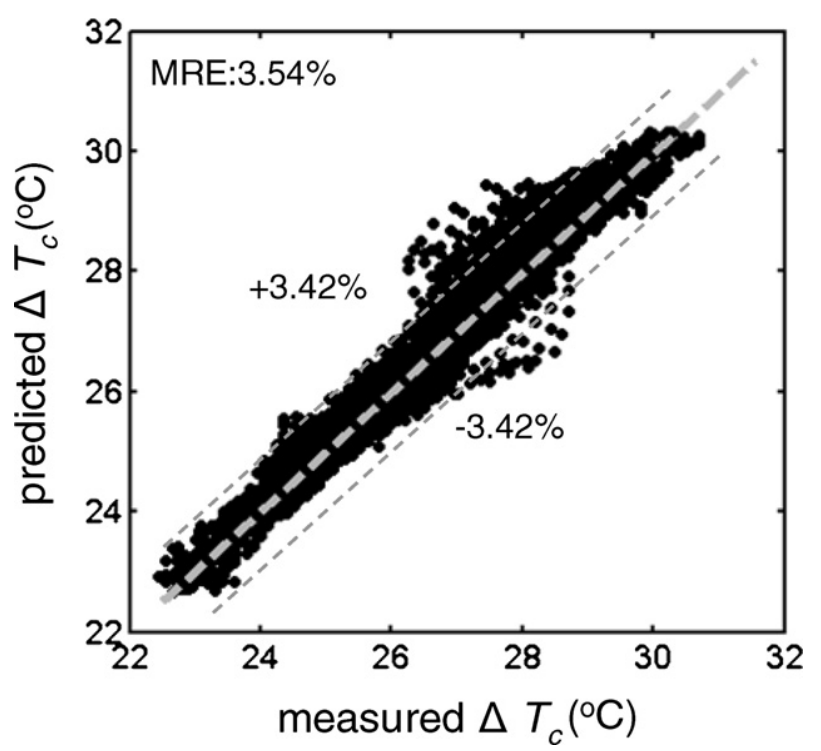

Fig. 17. Correlation between predicted results by the model predicted output (MPO) approach and measured results at $100 \mathrm{~W}$ heating power.

\section{Acknowledgement}

The authors deeply appreciate the financial support offered by the National Science Council (NSC) of Taiwan, Republic of China under contract NSC 94-2212-E-002-049.

\section{References}

[1] H. Akachi, Structure of a heat pipe, U.S. Patent No. 4921041, 1990.

[2] M. Hosoda, S. Nishio, R. Shirakashi, Study of meandering closed-loop heat transport device (vapor-plug propagation phenomena), JSME International Journal Series, B 42 (1999) 737-744.

[3] S.M. Lee, I.S. Kang, Three-dimensional analysis of the steady-state shape and small-amplitude oscillation of a bubble in uniform and non-uniform electric fields, Journal of Fluid Mechanics 384 (1999) 59-91.

[4] J.F. Yang, T. Ohji, Evaluation of mechanical properties of porous silicon nitride produced by partial hot-pressing, Ceramic Engineering and Science Proceedings 22 (2001) 243-250.

[5] S. Khandekar, P. Charoensawan, M. Groll, P. Terdtoon, Closed loop pulsating heat pipes part b: visualization and semi-empirical modeling, Applied Thermal Engineering 23 (2003) 2021-2033.

[6] H. Akachi, F. Polasek, P. Stulc, Pulsating heat pipes, in: Proceedings of the Fifth International Heat Pipe Symposium, Melbourne, Australia, 1996, 208217.

[7] G. Hung, L. Stark, The kernel identification method (1910-1977) - review of theory, calculation, application, and interpretation, Mathematical Biosciences 37 (1977) 135-190.

[8] S.A. Billings, W.S.F. Voon, Correlation based model validity tests for nonlinear models, International Journal of Control 44 (1986) 235-244.

[9] Z.V. Marmarelis, Practical identification of the general time-variant nonlinear dynamic system, Proceedings International Conference on Cybernetics and Society (1979) 727-733.

[10] M. Schetzen, Nonlinear system modeling based on the wiener theory, Proceedings of the IEEE 69 (1981) 1557-1573.

[11] M.J. Korenberg, S.B. Bruder, P.J. Mcllroy, Exact orthogonal kernel estimation from finite data records: extending wiener's identification of nonlinear systems, Annals of Biomedical Engineering 16 (1988) 201214.

[12] J.M. Korenberg, W.I. Hunter, Identification of nonlinear biological systems. Wiener Kernel approaches, Annals of Biomedical Engineering 18 (1990) 629654.

[13] G.K. Hung, L.W. Stark, Interpretation of kernels - an overview, Annals of Biomedical Engineering 19 (1991) 509-519.

[14] Z.V. Marmarelis, Error analysis and optimal estimation procedures in identification of nonlinear Volterra systems, Automatica 15 (1979) 161174.

[15] D.T. Westwick, R.E. Kearney, Nonlinear identification of the stretch reflex emg, Proceedings of the Annual Conference on Engineering in Medicine and Biology 15 (1993) 509-510.

[16] I.J. Leontaris, S.A. Billings, Input-output parametric models for non-linear systems, International Journal of Control 41 (1985) 311-341. 
[17] S.A. Billings, S. Chen, R.J. Backhouse, Identification of linear and non-linear models of a turbocharged automotive diesel engine, Mechanical Systems Signal Process 3 (1989) 123-142.

[18] J.J. Liu, Y.W. Lee, F.C. Wang, R. Uppala, P.H. Chen, Time and frequency domain identification and analysis of a permanent magnet synchronous servo motor, Journal of Chinese Institute of Engineers 29 (2006) 683-695.
[19] Y.W. Lee, Study on thermal performance and dynamic behavior of two-phase flow in miniature heat pipe, Ph.D. Thesis, Department of Mechanical Engineering, National Taiwan University, Taipei, 2006.

[20] K.M. Tsang, S.A. Billings, Two-dimensional pattern analysis and classification using a complex orthogonal estimation algorithm, IEE Proceedings: Vision, Image and Signal Processing 141 (1994) 339-347. 